pain syndrome. This study also showed that although antibiotic therapy is inappropriate and potentially harmful for such patients, as many of them receive antibiotics as do patients with infectious prostatitis.

In this study, the authors retrospectively screened US Veterans Health Administration inpatient, outpatient and pharmacy databases to identify men with a diagnosis of prostatitis. In the 2003 fiscal year, these databases included 4,758,039 men, of whom 28,124 had a diagnosis of prostatitis. Of these men, 23,037 (82\%) had chronic or unspecified prostatitis that was consistent with a diagnosis of chronic pelvic pain syndrome (overall annual prevalence $0.50 \%$ ). Among the men considered to have chronic pelvic pain syndrome, $69 \%$ had received an oral antibiotic prescription in 2003 compared with only $14 \%$ of the $4,626,221$ men who did not have chronic pelvic pain syndrome, infectious and/or acute prostatitis, or urinary-tract infections; $49 \%$ of men with chronic pelvic pain syndrome had received fluoroquinolone. After adjusting for confounding variables, the prevalence of fluoroquinolone prescription was similar in men with chronic pelvic pain syndrome and in those with infectious and/or acute prostatitis.

Original article Taylor BC et al. (2008) Excessive antibiotic use in men with prostatitis. Am J Med 121: 444-449

\section{Treating functional urinary incontinence can improve problem behavior in children}

In children, non-neuropathic bladder-sphincter dysfunction is associated with behavioral problems, but whether a cause and effect relationship exists is unclear. To investigate, Bael and colleagues conducted a prospective study to determine the prevalence of behavioral problems in children with functional urinary incontinence before and after incontinence treatment, and also assessed whether behavioral problems influenced treatment outcome.

The study included 202 children aged 6-12 years (97 with urge syndrome and 105 with dysfunctional voiding) enrolled in a European, randomized controlled trial of incontinence treatment. Parents or caregivers rated their child's behavior by completing Achenbach's Child Behavior Checklist at entry (scores available for 188 children) and 1 year after treatment completion (111 scores available).

Before treatment, $19 \%$ of the children had an abnormal total behavioral problem scoretwice the rate for a normative population - but this rate dropped to $11 \%$ after treatment. Interestingly, this drop largely reflected a significant reduction in the prevalence of externalization problems (aggressive or delinquent behavior) in children with dysfunctional voiding. By contrast, the prevalence of internalization problems (withdrawn behavior, depression, etc.) in both functional subgroups and that of externalization problems in the urge-syndrome subgroup did not fall significantly. Distribution of treatment outcomes (cured, no change, or worse) was the same for children with an initial abnormal total behavioral problem score as for the overall cohort.

Although behavioral problems did not influence the outcome of incontinence treatment, treatment for dysfunctional voiding did reduce the prevalence of externalization problems.

Original article Bael A et al. (2008) Behavior profiles in children with functional urinary incontinence before and after incontinence treatment. Pediatrics 121: e1196-e1200 